\title{
COMPARISON BETWEEN DIETARY INTAKE AND AGE IN RELATION TO BODY MASS INDEX AMONG POLYCYSTIC OVARIAN SYNDROME WOMEN - A CASE-CONTROL STUDY
}

\author{
BRINDHA G*, MADHANSHANKAR SR \\ Department of Biotechnology, PSG College of Arts and Science, Bharathiar University, Coimbatore, Tamil Nadu, India. \\ Email: brindhaganesan14@gmail.com
}

Received: 03 March 2019, Revised and Accepted: 28 May 2019

\section{ABSTRACT}

Objective: Polycystic ovarian syndrome (PCOS) is the most prevalent endocrine disorder, affecting up to $6.8 \%$ of women at their prime reproductive age. The aim of the study is to prove the interrelationship between food patterns, age, and anthropometric measurements in relation with body mass index (BMI) among PCOS women and control participants.

Methods: The study was performed among 80 PCOS women and control participants according to the Rotterdam criteria. The data were collected through questionnaire which included age, socioeconomic data, anthropometric details, and food pattern, and the values were analyzed using Minitab 17 by Box plot method for graphical representation. The comparative analysis between age group and BMI was done by ANOVA.

Results: The results showed that there was significant relationship between inappropriate food pattern and PCOS women showing higher frequency of \pm 8.30 among age group (19-24 years) with an average BMI of $31.6 \mathrm{~kg} / \mathrm{m}^{2}$. The unhealthy diet along with BMI was correlated with the visual examination of presence of hirsutism. From the ANOVA analysis, it was significantly proved with probability ( $\mathrm{p}=0.002$ ); there was stronger association between lifestyle modifications and PCOS. This is the first association study to correlate between dietary habitat and age group with BMI among South Indian in Coimbatore district.

Conclusion: From the results obtained, it is necessary to create awareness for women and girls about the ill effects of lifestyle modifications, unhealthy food pattern, and lack of physical activity which lead hormonal and metabolic changes.

Keywords: Polycystic ovarian syndrome, Hirsutism, Body mass index, Hyperandrogenism, Anthropometric details.

(C) 2019 The Authors. Published by Innovare Academic Sciences Pvt Ltd. This is an open access article under the CC BY license (http://creativecommons. org/licenses/by/4. 0/) DOI: http://dx.doi.org/10.22159/ajpcr.2019.v12i7.33917

\section{INTRODUCTION}

Polycystic ovarian syndrome (PCOS) is a complex heterogeneous chronic clinical condition characterized by menorrhagia hyperandrogenism and polycystic ovaries [1]. Anxiety, depression, psychological disturbances, metabolic derangements, poor selfesteem, strained relationships, decreased quality of life, disordered eating pattern, and psychosexual dysfunction are the implications of the syndrome [2]. PCOS is also defined as oligogenic disorder which is associated with the interaction of a number of genetic and environmental factors that influences the heterogeneous, clinical, and biochemical phenotype [3]. The prevalence of PCOS has been reported about $5-10 \%$ worldwide, which varies between population arising from genetic factors and also sudden shift to westernized diets and sedentary lifestyle among Asians. India is the second most leading country in the world with about 1.2 billion people and is emerging with rapid epidemiological transition [4]. Women with PCOS have an elevated level of body mass index (BMI) which increases the pathophysiological factors such as insulin resistance and hyperandrogenism through augmenting ovarian androgen secretion [5]. Hirsutism affects up to $60-80 \%$ of women and this severity is related to sensitivity of hair follicles to androgens [6]. Hirsutism is a common manifestation of hyperandrogenism due to the elevated levels of circulating androgen apart the other signs of excess secretion of androgen including acne, seborrhea, androgenic alopecia, and also virilization. Environmental and genetic factors implicated in PCOS mainly can be exacerbated by lack of physical activity, packed food beverages, infectious agents, and fast foods play a major role. The etiology of PCOS remains unknown but due to various predisposing factors that interact with environmental and lifestyle aspects yet PCOS is considered as complex genetic disorder. There is a paucity of research which details the effect of lifestyle and morphological factors and their interlink responsible for the onset of PCOS. As PCOS incidence is on rise in India, it is pertinent to create awareness among people about the condition and its consequences. The evaluation of covariation between dietary pattern who has sedentary lifestyle and influence of fast-growing westernized diet in relation with hirsutism along with their age factor among South Indian women has not yet been studied completely. This study makes an attempt to quantify the effect and relationship between life style habitat, age, BMI, and anthropometric characteristics of PCOS diagnosed women in relation to BMI.

\section{METHODS}

Ethical approval

The study was conducted with the approval of the Institutional Human Ethics Committee Human Ethical clearance at PSG Institute of Medical Science and Research July 2017 (ref no: 15/133). This study was a randomized observational study carried at the Gynecology Department, Women's Center by Motherhood, Coimbatore, for a period of 6 months. The PCOS diagnosed women with an age group of 18-30 years were taken for investigation. Screening of PCOS participants was diagnosed based on Rotterdam consensus criteria: Two of the three criteria are necessary for diagnosis - (1) oligo or anovulation, (2) clinical and/or biochemical signs of hyperandrogenism, and (3) presence of polycystic ovaries. Before further study, all consenting women were appraised about the study, and a consent letter both in English and vernacular language (Tamil) was obtained.

Sample collection criteria

About 80 patients were selected based on inclusion and exclusion criteria. 
Inclusion criteria

Oligomenorrhea or amenorrhea, clinical hyperandrogenism, and formation of polycystic ovaries were included from the study.

\section{Exclusion criteria}

All patients with diabetes mellitus, hypertension, hyperprolactinemia, thyroid disorder, Cushing's syndrome, premature ovarian failure, ovarian tumors, and usage of oral contraceptive pill use within the past 6 months were excluded from the study.

All consenting women subjected in this study were followed with a self-administered questionnaire which includes their age, socioeconomic data, anthropometric measurements, food habit, and morphological status. The details were acquired under respective conditions: The presence of polycystic ovaries was confirmed by transabdominal ultrasonography. The body weight was measured without shoes with a portable stadiometer scale. Their BMI was calculated with measured weight and height data along with visual examination and the presence of hirsutism was examined. The food habit details were obtained through the questionnaire as it comprises the healthy food which includes intake of fruits, vegetables, and fresh juices and unhealthy dietary pattern comprises consumption of nonvegetarian, carbonated drinks, and fast food such as pizza, burger, and fried foods.

\section{RESULTS}

A total of 80 participants were enrolled in this study. The information collected through questionnaire was tabulated under different categories as age, BMI, food pattern, and hirsutism. These values were statistically interpreted by statistical software: Minitab 17 by Box plot method for graphical representation. Box plot is an exploratory

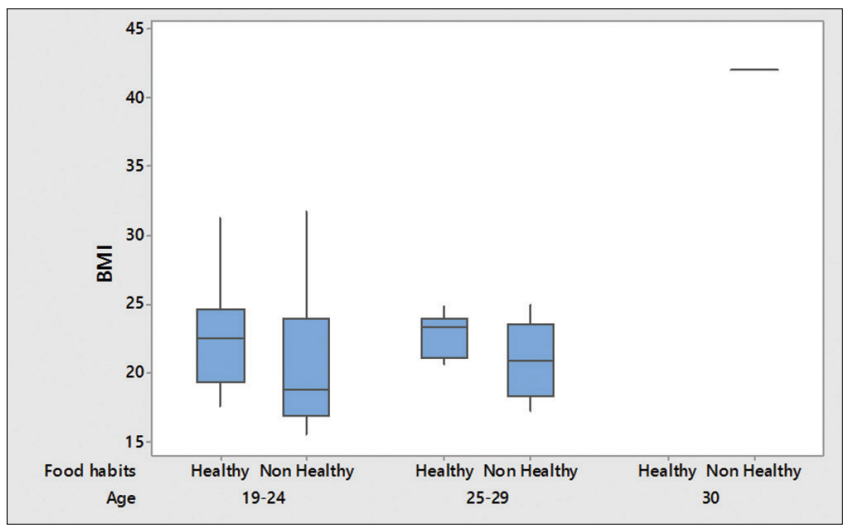

Fig. 1: Graphical representation of control participants body mass index with age and food habit

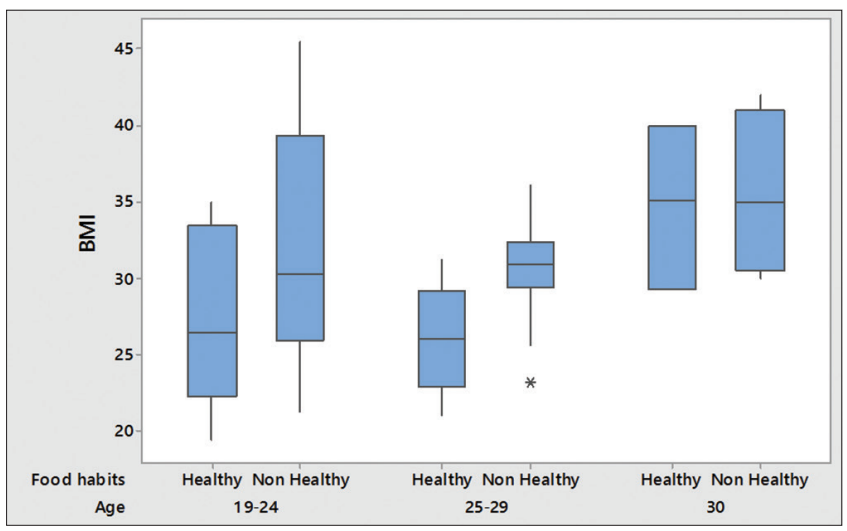

Fig. 2: Graphical representation of polycystic ovarian syndrome women body mass index with age and food habit graphic method used to show the distribution of a dataset. The values were segregated, and graphical representation was done. Comparative graphical representation was plotted between BMI and age group years by analysis of variance (ANOVA) for both the participants.

From the tabulated results (Table 1), standard deviation frequency was done for different age group - (19-24) adolescence age group, (25-29) adult women, and above 30 years (Figs. 1 and 2) along with frequency of food patterns - differentiating as healthy and unhealthy food and having BMI as major factor for both control and PCOS women. The age group between 19 and 24 years had higher frequency of \pm 8.30 of BMI in comparative with other age groups, and furthermore, it depicts that due to unhealthy food habit, the women in age group 19-24 years, the average BMI is $31.6 \mathrm{~kg} / \mathrm{m}^{2}$ and for 30 years and above, it is $37.55 \mathrm{~kg} / \mathrm{m}^{2}$ than that of non-PCOS women. From the entire age group category, the SD value of BMI was high than that of control participants.

The result from Table 2 depicts that hyperandrogenism-excess secretion of androgen plays significant part in PCOS women in relation with age and BMI. From the above results, comparative analysis was done between BMI and hirsutism along with age factor (Fig. 3), which depicted that due to unhealthy food habit, there was disruption in endocrine receptors which lead to excess secretion of androgen which was absent in control participants. The presence of hirsutism was noted with a higher frequency of \pm 8.13 with an average BMI of 30.24 $\left(\mathrm{kg} / \mathrm{m}^{2}\right)$ among age group 19-24 (Fig. 4) years at higher incidence level.

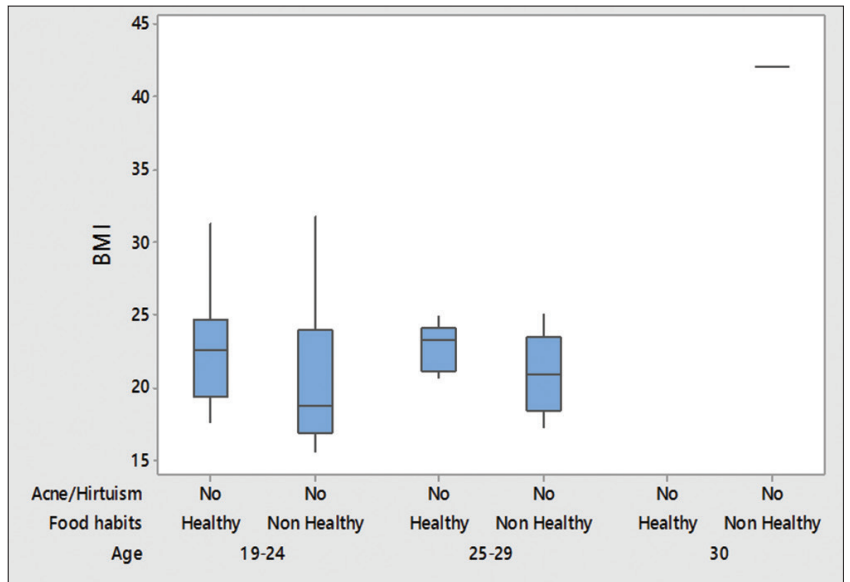

Fig. 3: Representation of morphological characteristic of nonpolycystic ovarian syndrome subjects in relation with age, food pattern with body mass index

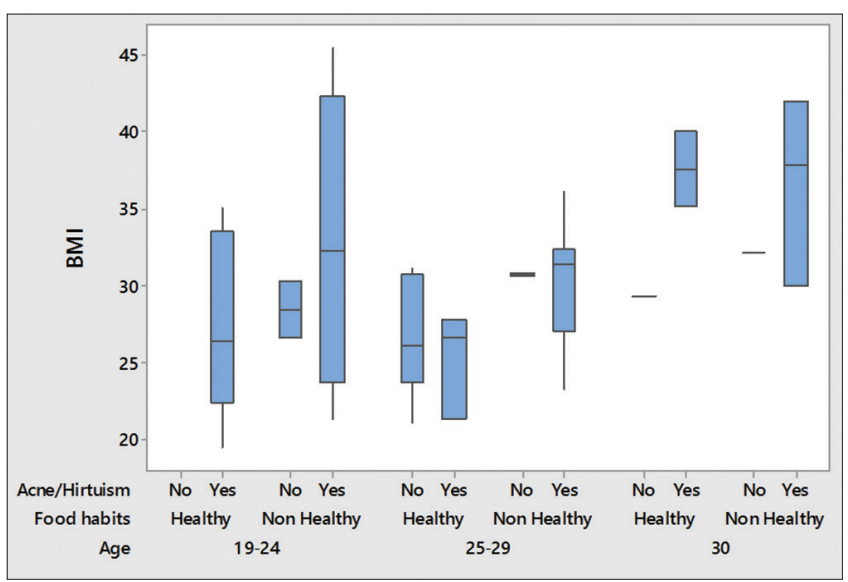

Fig. 4: Comparison of visual examination of hirsutism along with different age category and body mass index 
Table 1: Comparative analysis of BMI dataset between age and food habits among PCOS and non-PCOS women

\begin{tabular}{|c|c|c|c|c|c|}
\hline \multicolumn{2}{|l|}{ Parameters } & \multicolumn{2}{|l|}{ Control } & \multicolumn{2}{|c|}{$\begin{array}{l}\text { PCOS diagnosed } \\
\text { women }\end{array}$} \\
\hline & & \multicolumn{2}{|l|}{ BMI } & \multicolumn{2}{|l|}{ BMI } \\
\hline Age (years) & Food habit & Average & SD & Average & SD \\
\hline $19-24$ & Un health & 20.9 & \pm 5.3 & 31.6 & \pm 8.30 \\
\hline $19-24$ & Healthy & 22.55 & \pm 3.48 & 27.63 & \pm 6.06 \\
\hline $25-29$ & Un healthy & 20.94 & \pm 2.9 & 30.42 & \pm 3.60 \\
\hline $25-29$ & Healthy & 22.85 & \pm 1.6 & 25.22 & \pm 3.53 \\
\hline 30 & Un healthy & $\begin{array}{l}\text { Only one } \\
\text { data (BM }\end{array}$ & Imple & 35.5 & \pm 5.47 \\
\hline 30 & Healthy & - & & 33.55 & \pm 5.37 \\
\hline
\end{tabular}

BMI: Body mass index, PCOS: Polycystic ovarian syndrome

Table 2: Comparative tabulation between BMI and Hirsutism based on age factor

\begin{tabular}{|c|c|c|c|c|c|}
\hline \multirow{2}{*}{\multicolumn{2}{|c|}{ Parameters }} & \multicolumn{2}{|c|}{ Non-PCOS } & \multicolumn{2}{|l|}{ PCOS } \\
\hline & & \multicolumn{2}{|l|}{ BMI } & \multicolumn{2}{|l|}{ BMI } \\
\hline Age & Hirsutism & Average & SD & Average & SD \\
\hline $19-24$ & Yes & - & - & 30.24 & \pm 8.13 \\
\hline $19-24$ & No & 21.86 & \pm 4.31 & 28.47 & \pm 2.64 \\
\hline $25-29$ & Yes & - & - & 29 & \pm 4.46 \\
\hline $25-29$ & No & 21.82 & \pm 2.5 & 27.63 & \pm 3.74 \\
\hline 30 & Yes & - & - & 37 & \pm 4.67 \\
\hline 30 & No & \multicolumn{2}{|c|}{$\begin{array}{l}\text { Only one sample } \\
\text { data (BMI 42) }\end{array}$} & 30.7 & \pm 2.00 \\
\hline
\end{tabular}

BMI: Body mass index, PCOS: Polycystic ovarian syndrome

\section{DISCUSSION}

In the present era of modernization and adoption of convenient ways of westernized living, India has seen an upsurge among the women with PCOS. From this study, we have perceived that there is a drastic lifestyle modification where people have changed from conventional to convenience food habitat and also lack of physical activity and have adopted themselves to sedentary lifestyle pattern at young age.

From the obtained data, the segregation was under three different age category, in which 19-24 years of girls of their prime reproductive age have higher BMI of $45.4\left(\mathrm{~kg} / \mathrm{m}^{2}\right)$ with an average of $31.6 \%$ which was predominantly higher in comparison to other groups; similarly, Eleftheriadou et al. ${ }^{[7]}$ have made remarkable observation that BMI is interrelated to PCOS. Although control participants consumed unhealthy food, their BMI and average frequency were much lesser compared to that of PCOS women. The BMI values were considered $18-25\left(\mathrm{~kg} / \mathrm{m}^{2}\right)$ as normal weight, $25-30 \mathrm{~kg} / \mathrm{m}^{2}$ as overweight, and $>30 \mathrm{~kg} / \mathrm{m}^{2}$ as obese women. From this analysis, it was reported that with $95 \%$ confidence interval, $50 \%$ of PCOS women were found to be obese and similarly $25 \%$ were overweight. The box plot reveals a higher inclination toward outset of obesity in the PCOS diagnosed women which is incident due to a higher BMI. Diet and nutritional habits are most important factors in life which affects our physical health. In our study, it was reported that there was diverse change in consumption of food items such as soft drinks, fast food, lack of fruits, and vegetables among PCOS women when compared with control participants. This finding of dietary pattern prone to cause psychological distress, menstrual disturbances, affects metabolic signals, hormonal imbalance, and infertility. PCOS patients with higher BMI are prone to suffer hyperandrogenism or to exhibit clinical symptoms of excess androgen secretion [8]

Existing study revealed the importance of BMI as a prognostic factor for various biological and morphological aspects. As a part of physical examination, our findings highlighted the significance of evaluating BMI

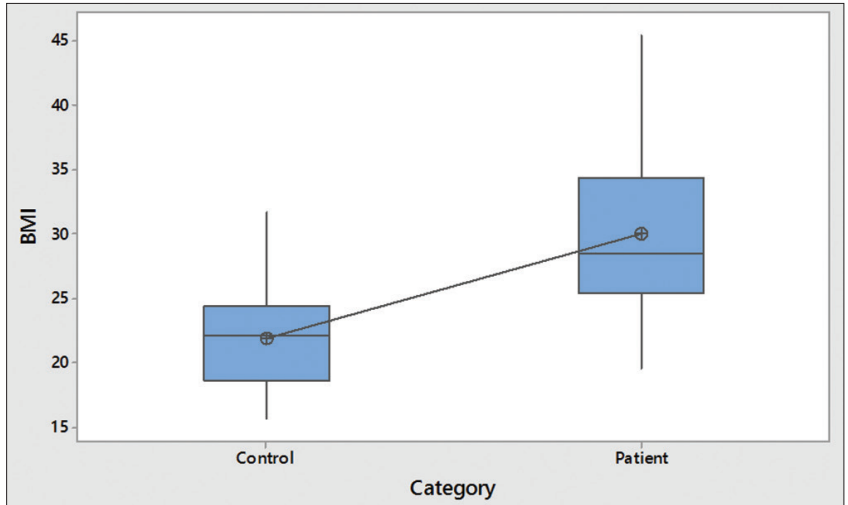

Fig. 5: Comparative graph: BMI and age group

in relation to hirsutism. Based on the age group 19-24 years and above 30 years showed an average of 30.24 and 37 with standard deviation of \pm 8.13 and \pm 4.67 , respectively, where people with higher BMI are more likely to suffer from hyperandrogenism or expose clinical signs of excess secretion of androgen.

Of the PCOS diagnosed women, 19 reported that they consumed fast food, fried items twice in a week, 12 had once in a week, and 9 consume very rarely such food. From the above comparative results, the probability ( $\mathrm{p}=0.002$ ) (Fig. 5) shows that there is a significant association between age and BMI, especially at the age group of 19-24 at prime reproductive age, the prevalence rate of PCOS is higher.

This is the first association study to interpret lifestyle modifications and food habits shall have a direct relationship to the onset and progression of PCOS in relation to age and BMI where higher BMI will lead to excess secretion of androgen among women at younger age among South Indian population in Coimbatore. Studies have proved that lifestyle modifications and balanced diet are first-line treatment for women with PCOS. Physical and mental health aspects with proper medication will be the first-line treatment for PCOS [9]. Pharmacological therapy also paves way for the treatment of PCOS that improves hyperandrogenism along with insulin sensitivity which assists regular menstruation and fertility problems and also prevents endocrine and metabolic consequences [10]. The outcome of this study has attached that the ill effects of sedentary lifestyle changes and lack of physical exercises would cause psychological distress, reproductive and metabolic abnormalities, and cardiovascular diseases among women.

\section{CONCLUSION}

The onset of diseases like PCOS proves to be a serious condition severely hampering the reproduction potentials of women. It is important for health-care providers to understand the pathophysiology of PCOS and also exact evaluation of this disorder to rule out underlying disease. Early diagnosis and intervention will reduce the long-term health complications associated with PCOS. Hence, the management of PCOS must include a lifestyle intervention which improves clinical features, body composition, and metabolism in women with PCOS. Since PCOS is not just confined to menstrual and fertility issues and since major metabolic disturbances are also possible in the affected individual with long-standing impact on health, this type of study can serve as a possible launch pad for defining long-term therapies.

\section{ACKNOWLEDGMENT}

The authors are extremely grateful to Dr. Mirudhubashini Govindarajan, Obstetrician and Gynecologist, Women's Center by Motherhood, Coimbatore, for her constant support and help in carrying out this study.

\section{AUTHORS' CONTRIBUTIONS}

All authors have made great contribution to this work. 


\section{CONFLICTS OF INTEREST}

The authors declare that they have no conflicts of interest.

\section{REFERENCES}

1. Rosenfield RL, Ehrmann DA. The pathogenesis of polycystic ovary syndrome (PCOS): The hypothesis of PCOS as functional ovarian hyperandrogenism revisited. Endocr Rev 2016;37:467-520.

2. Xita N, Georgiou I, Tsatsoulis A. The genetic basis of polycystic ovary syndrome. Eur J Endocrinol 2002;147:717-25.

3. Pradeepa R, Anjana RM, Joshi SR, Bhansali A, Deepa M, Joshi PP, et al. Prevalence of generalized and abdominal obesity in urban and rural India. Indian J Med Res 2015;142:139.

4. Lim SS, Norman RJ, Davies MJ, Moran LJ. The effect of obesity on polycystic ovary syndrome: A systematic review and meta-analysis. Obes Rev 2013;14:95-109.

5. Albu A, Radian S, Fica S, Barbu CG. Biochemical hyperandrogenism is associated with metabolic syndrome independently of adiposity and insulin resistance in Romanian polycystic ovary syndrome patients. Endocrine 2015;48:696-704

6. Fauser B, Tarlatzis B, Rebar RW, Legro RS, Balen AH, Lobo R, et al. Consensus on women's health aspects of polycystic ovary syndrome (PCOS): The Amsterdam ESHRE/ASRM-sponsored $3^{\text {rd }}$ PCOS consensus workshop group. Fertil Steril 2012;97:28-38. e25.

7. Shahedur R, Anowar P, Abdus S, Shahjahan A. study of the effect of food habit, lifestyle and daily trip on physical and mental status of subjects at Islamic university in Kushtia, Bangladesh. Open J Stat 2012;2:219-23

8. Yuan C, Liu X, Mao Y, Diao F, Cui Y, Liu J. Polycystic ovary syndrome patients with high BMI tend to have functional disorders of androgen excess: A prospective study. J Biomed Res 2016;30:197-202.

9. Patil VR, Thangavelu PD, Jagtap VK. Effectiveness of lifestyle modification on weight loss and quality of life in obese women with polycystic ovarian syndrome Asian J Pharm Clin Res 2018;11:333-6.

10. Muhas C, Nishad KM, Ummunnoora KP, Jushna K, Saheera KV, Dilsha KP. Polycystic ovary syndrome (PCOS) an overview. Int J Curr Pharm Res 2018;10:5-9. 